\title{
Article \\ Comprehensive Immunohistochemical Study of the SWI/SNF Complex Expression Status in Gastric Cancer Reveals an Adverse Prognosis of SWI/SNF Deficiency in Genomically Stable Gastric Carcinomas
}

\author{
Marie-Isabelle Glückstein ${ }^{1}$, Sebastian Dintner ${ }^{1}$, Tim Tobias Arndt ${ }^{1,2}{ }^{(D}$, Dmytro Vlasenko ${ }^{3}$, \\ Gerhard Schenkirsch ${ }^{4}$, Abbas Agaimy ${ }^{5}{ }^{\circledR}$, Gernot Müller ${ }^{2}$, Bruno Märk1 ${ }^{1}{ }^{\mathbb{D}}$ and Bianca Grosser ${ }^{1, *}$
}

1 Institute of General Pathology and Molecular Diagnostics, University Hospital Augsburg, 86156 Augsburg, Germany; Marie-Isabelle.Glueckstein@uk-augsburg.de (M.-I.G.); sebastian.dintner@uk-augsburg.de (S.D.); tobias.arndt@uka-science.de (T.T.A.); bruno.maerkl@uka-science.de (B.M.)

2 Institute of Mathematics and Computational Statistics, University of Augsburg, 86159 Augsburg, Germany; gernot.mueller@math.uni-augsburg.de

3 Department of General, Visceral and Transplantation Surgery, University Hospital Augsburg, 86156 Augsburg, Germany; dmytro.vlasenko@uk-augsburg.de

check for updates

Citation: Glückstein, M.-I.; Dintner,

S.; Arndt, T.T.; Vlasenko, D.;

Schenkirsch, G.; Agaimy, A.; Müller,

G.; Märkl, B.; Grosser, B.

Comprehensive Immunohistochemical

Study of the SWI/SNF Complex

Expression Status in Gastric Cancer

Reveals an Adverse Prognosis of

SWI/SNF Deficiency in Genomically

Stable Gastric Carcinomas. Cancers

2021, 13, 3894. https://doi.org/

10.3390/cancers13153894

Academic Editors: Lucia Magnelli and Yoshinobu Hirose

Received: 15 July 2021

Accepted: 30 July 2021

Published: 2 August 2021

Publisher's Note: MDPI stays neutral with regard to jurisdictional claims in published maps and institutional affiliations.

Copyright: (c) 2021 by the authors. Licensee MDPI, Basel, Switzerland. This article is an open access article distributed under the terms and conditions of the Creative Commons Attribution (CC BY) license (https:// creativecommons.org/licenses/by/ $4.0 /)$.
4 Tumor Data Management, University Hospital Augsburg, 86156 Augsburg, Germany; Gerhard.Schenkirsch@uk-augsburg.de

5 Institute of Pathology, Friedrich-Alexander-University Erlangen-Nürnberg, University Hospital Erlangen, 91054 Erlangen, Germany; Abbas.Agaimy@uk-erlangen.de

* Correspondence: bianca.grosser@uk-augsburg.de; Tel.: +49-821-400-2150; Fax: +49-821-400-16-2150

Simple Summary: This study aimed to investigate the clinical relevance of immunohistochemical expression of proteins of the SWI/SNF complex, SMARCA2, SMARCA4 SMARCB1, ARID1A, ARID1B, and PBRM1 in 477 adenocarcinomas of the stomach and gastroesophageal junction. Additionally, the tumors were classified immunohistochemically in analogy to The Cancer Genome Atlas (TCGA) classification. Overall, $32 \%$ of cases demonstrated aberrant expression of the SWI/SNF complex. SWI/SNF aberration emerged as an independent negative prognostic factor for overall survival in all patients and in genomically stable patients in analogy to TCGA. In conclusion, determination of SWI/SNF status could be suggested in routine diagnostics in genomically stable tumors to identify patients who might benefit from new therapeutic options.

Abstract: The SWI/SNF complex has important functions in the mobilization of nucleosomes and consequently influences gene expression. Numerous studies have demonstrated that mutations or deficiency of one or more subunits can have an oncogenic effect and influence the development, progression, and eventual therapy resistance of tumor diseases. Genes encoding subunits of the SWI/SNF complex are mutated in approximately $20 \%$ of all human tumors. This study aimed to investigate the frequency, association with clinicopathological characteristics, and prognosis of immunohistochemical expression of proteins of the SWI/SNF complexes, SMARCA2, SMARCA4 SMARCB1, ARID1A, ARID1B, and PBRM1 in 477 adenocarcinomas of the stomach and gastroesophageal junction. Additionally, the tumors were classified immunohistochemically in analogy to The Cancer Genome Atlas (TCGA) classification. Overall, 32\% of cases demonstrated aberrant expression of the SWI/SNF complex. Complete loss of SMARCA4 was detected in three cases $(0.6 \%)$ and was associated with adverse clinical characteristics. SWI/SNF aberration emerged as an independent negative prognostic factor for overall survival in genomically stable patients in analogy to TCGA. In conclusion, determination of SWI/SNF status could be suggested in routine diagnostics in genomically stable tumors to identify patients who might benefit from new therapeutic options.

Keywords: gastric cancer; SWI/SNF; immunohistochemistry 


\section{Introduction}

Gastric cancer is ranked as the sixth-most-common cancer entity worldwide, having accounted for approximately 780,000 cancer-associated deaths in 2018 [1]. So far, the best parameter for predicting prognosis and, therefore, therapy in gastric-cancer patients is TNM staging. The factors that are relevant for determining the prognosis of gastric carcinomas are local infiltration depth, locoregional lymph node involvement, distant metastases, and vascular invasion [2-4]. Additionally, diffuse Laurén subtype and proximal tumor localization are also known negative prognostic factors [5-7]. The introduction of perioperative chemotherapy after 2005 has improved the outcome in stage two and three gastric cancers, with a median survival of 50 months vs. 34 months [8]. However, the prognosis of gastric cancer is still poor and has a five-year-survival rate that has not changed during the period between 2000 and 2014, with survival rates being between $31.4 \%$ and $33.5 \%$ in Germany [9].

Two generally accepted molecular classifications have been proposed for gastric carcinomas, which have both prognostic and therapeutic implications, namely The Cancer Genome Atlas (TCGA) and the Asian Cancer Research Group (ACRG) classification [10,11]. So far, only a few prognostic and therapeutic biomarkers have been identified for gastric cancer. To date, the most important therapeutic marker in gastric carcinoma is HER2 overexpression [12]. In addition, MSI status and high PDL1 expression are independent positive prognostic factors in gastric carcinoma [13-15], while aberrant E-cadherin expression is considered an unfavorable prognostic factor and even a negative predictive factor for chemotherapy response [16].

SMARCA2, SMARCA4, SMARCB1, ARID1A, ARID1B, and PBRM1 are subunits of the Switch/Sucrose non-fermenting (SWI/SNF) complex, which show frequent alterations in rhabdoid tumors, ovarian clear cell carcinomas (OCCCs), and small-cell carcinoma of the ovary, hyper-calcemic type (SCCOHT) $[17,18]$. Numerous studies have demonstrated that this complex plays a role in tumor suppression in human cancers. Mutations or deficiencies of one or more subunits can have an oncogenic effect and influence the development, progression, and eventual therapy resistance of tumor diseases [18-20]. A few studies have also already demonstrated loss or heterogeneous expression patterns of these subunits in gastric carcinoma, which could make them potential starting points for new therapeutic concepts [21-25].

The aim of this retrospective study was to determine whether and to what extent molecular aberrations of SWI/SNF complex subunits play a role in gastric cancer in a large Western cohort. For this purpose, we evaluated the frequency, association with clinicopathological characteristics, and prognosis of alterations in SMARCA2, SMARCA4, SMARCB1, ARID1A, ARID1B, and PBRM1 in 477 carcinomas of the stomach and gastroesophageal junction. In addition, association with the subgroups of the molecular TCGA classification was investigated. Furthermore, determination of SWI/SNF status was reduced to SMARCA2, SMARCA4, SMARCB1, and ARID1A expression to facilitate applicability in routine diagnostics.

\section{Materials and Methods}

\subsection{Patients}

Surgical resection specimens from 511 patients with adenocarcinomas of the stomach and the gastroesophageal junction that were treated between 2005 and 2018 at the Department of Visceral Surgery of the University Hospital Augsburg were included in the study (AEGII and III according to Siewert and Stein [26]). Tumors from 34 patients were excluded from the study, because of low tumor percentage on the tissue microarray (TMA) and the final cohort consisted of 477 tumors. Of these, 347 tumors were treated with surgery alone, and 130 patients received neoadjuvant chemotherapy. Detailed clinical characteristics are summarized in Table 1. 
Table 1. Clinocopathological characteristics and SWI/SNF status.

\begin{tabular}{|c|c|c|c|c|c|c|c|}
\hline Variable & \multicolumn{2}{|c|}{$n=477^{*}$} & \multicolumn{2}{|c|}{$\begin{array}{l}\text { SWI/SNF-Aberrant } \\
\quad(n=153)\end{array}$} & \multicolumn{2}{|c|}{$\begin{array}{l}\text { SWI/SNF Intact } \\
\quad(n=319)\end{array}$} & $p$ Value \\
\hline Median age (range) (years) & \multicolumn{2}{|c|}{$70.0(30.0-95.0)$} & \multicolumn{2}{|c|}{$72.0(41.0-95.0)$} & \multicolumn{2}{|c|}{$68.0(30.0-94.0)$} & \multirow{2}{*}{0.193} \\
\hline Median survival (range) (months) & \multicolumn{2}{|c|}{$58.0(49.9-66.1)$} & \multicolumn{2}{|c|}{$54.0(34.7-73.3)$} & \multicolumn{2}{|c|}{$60.0(53.5-66.5)$} & \\
\hline \multicolumn{8}{|l|}{ Sex } \\
\hline Female & 165 & $35 \%$ & 61 & $40 \%$ & 104 & $33 \%$ & 0.121 \\
\hline Male & 312 & $65 \%$ & 92 & $60 \%$ & 215 & $67 \%$ & \\
\hline \multicolumn{8}{|l|}{ T status } \\
\hline $\mathrm{pT} 1 / 2$ & 159 & $33 \%$ & 38 & $25 \%$ & 121 & $38 \%$ & 0.005 \\
\hline $\mathrm{pT} 2 / 3$ & 318 & $67 \%$ & 115 & $75 \%$ & 198 & $62 \%$ & \\
\hline \multicolumn{8}{|l|}{$\mathrm{N}$ status } \\
\hline Negative & 178 & $37 \%$ & 53 & $35 \%$ & 122 & $38 \%$ & 0.448 \\
\hline Positive & 299 & $63 \%$ & 100 & $65 \%$ & 197 & $62 \%$ & \\
\hline \multicolumn{8}{|l|}{ Distant Metastasis } \\
\hline No & 247 & $52 \%$ & 75 & $49 \%$ & 171 & $54 \%$ & \multirow{3}{*}{0.409} \\
\hline Yes & 197 & $41 \%$ & 66 & $43 \%$ & 127 & $40 \%$ & \\
\hline NA & 33 & $7 \%$ & 12 & $8 \%$ & 21 & $7 \%$ & \\
\hline \multicolumn{8}{|l|}{ Grading } \\
\hline Low grade & 162 & $34 \%$ & 48 & $31 \%$ & 114 & $36 \%$ & \multirow{3}{*}{0.471} \\
\hline High grade & 304 & $64 \%$ & 100 & $65 \%$ & 204 & $64 \%$ & \\
\hline NA & 11 & $2 \%$ & 5 & $3 \%$ & 1 & $0 \%$ & \\
\hline \multicolumn{8}{|l|}{ Lymphovascular invasion } \\
\hline Negative & 287 & $60 \%$ & 85 & $56 \%$ & 199 & $62 \%$ & \multirow[t]{2}{*}{0.156} \\
\hline Positive & 190 & $40 \%$ & 68 & $44 \%$ & 120 & $38 \%$ & \\
\hline \multicolumn{8}{|l|}{ Vascular invasion } \\
\hline Negative & 401 & $84 \%$ & 128 & $84 \%$ & 268 & $84 \%$ & \multirow[t]{2}{*}{0.922} \\
\hline Positive & 76 & $16 \%$ & 25 & $16 \%$ & 51 & $16 \%$ & \\
\hline Laurén & & & & & & & 0.159 \\
\hline Intestinal & 266 & $56 \%$ & 93 & $61 \%$ & 172 & $54 \%$ & \\
\hline Non-intestinal & 211 & $44 \%$ & 60 & $39 \%$ & 147 & $46 \%$ & \\
\hline Localization & & & & & & & \\
\hline Proximal & 124 & $26 \%$ & 36 & $24 \%$ & 85 & $27 \%$ & \\
\hline Non-proximal & 335 & $70 \%$ & 111 & $73 \%$ & 222 & $70 \%$ & 0.471 \\
\hline NA & 18 & $4 \%$ & 6 & $4 \%$ & 12 & $4 \%$ & \\
\hline R status & & & & & & & \\
\hline R0 & 403 & $84 \%$ & 131 & $86 \%$ & 268 & $84 \%$ & \\
\hline $\mathrm{R} 1$ & 54 & $11 \%$ & 15 & $10 \%$ & 38 & $12 \%$ & 0.508 \\
\hline $\mathrm{Rx}$ & 20 & $4 \%$ & 7 & $5 \%$ & 13 & $4 \%$ & \\
\hline TCGA & & & & & & & \\
\hline $\mathrm{EBV}^{+}$ & 25 & $5 \%$ & 14 & $9 \%$ & 11 & $3 \%$ & \\
\hline MSI & 61 & $13 \%$ & 40 & $26 \%$ & 21 & $7 \%$ & \\
\hline GS & 110 & $23 \%$ & 29 & $19 \%$ & 79 & $25 \%$ & $<0.001$ \\
\hline $\mathrm{CIN}$ & 151 & $32 \%$ & 27 & $18 \%$ & 123 & $39 \%$ & \\
\hline No classification & 130 & $27 \%$ & 43 & $28 \%$ & 85 & $27 \%$ & \\
\hline Death & & & & & & & \\
\hline No & 227 & $48 \%$ & 67 & $44 \%$ & 159 & $50 \%$ & 0.218 \\
\hline Yes & 250 & $52 \%$ & 86 & $56 \%$ & 160 & $50 \%$ & \\
\hline nCTx & & & & & & & \\
\hline No & 347 & $73 \%$ & 112 & $73 \%$ & 234 & $73 \%$ & 0.972 \\
\hline Yes & 130 & $27 \%$ & 41 & $27 \%$ & 85 & $27 \%$ & \\
\hline
\end{tabular}


Table 1. Cont.

\begin{tabular}{|c|c|c|c|c|c|c|c|}
\hline Variable & \multicolumn{2}{|c|}{$n=477^{*}$} & \multicolumn{2}{|c|}{$\begin{array}{l}\text { SWI/SNF-Aberrant } \\
\quad(n=153)\end{array}$} & \multicolumn{2}{|c|}{$\begin{array}{c}\text { SWI/SNF Intact } \\
(n=319)\end{array}$} & \multirow[t]{2}{*}{$p$ Value } \\
\hline TRG & $\left(\begin{array}{c}n=130 \\
* * \\
*\end{array}\right)$ & & $(n=41)$ & & $(n=85$ & & \\
\hline $1 b$ & 10 & $7 \%$ & 0 & $0 \%$ & 9 & $11 \%$ & \multirow[t]{3}{*}{0.023} \\
\hline 2 & 37 & $28 \%$ & 9 & $22 \%$ & 28 & $33 \%$ & \\
\hline 3 & 83 & $63 \%$ & 32 & $78 \%$ & 48 & $56 \%$ & \\
\hline \multicolumn{7}{|l|}{ CTx regimen } & \multirow{6}{*}{0.142} \\
\hline $\mathrm{Cis} / \mathrm{Ox}+5-\mathrm{FU}$ or Cap & 36 & $27 \%$ & 14 & $34 \%$ & 20 & $24 \%$ & \\
\hline $\mathrm{Ox}+5-\mathrm{FU}+\mathrm{Doc}$ & 46 & $35 \%$ & 11 & $27 \%$ & 34 & $40 \%$ & \\
\hline Cis + 5-FU + Epi & 41 & $32 \%$ & 12 & $29 \%$ & 28 & $33 \%$ & \\
\hline $\mathrm{Ox}+\mathrm{Epi}+\mathrm{Cap}$ & 5 & $4 \%$ & 2 & $5 \%$ & 3 & $4 \%$ & \\
\hline Others & 2 & $2 \%$ & 2 & $5 \%$ & 0 & $0 \%$ & \\
\hline
\end{tabular}

p-values of $\mathrm{Chi}^{2}$-test are shown for difference between SWI/SNF aberrant and intact tumors. NA, not available; TCGA, The Cancer Genome Atlas; EBV ${ }^{+}$, EBV-positive; MSI, microsatellite instable; GS, genomically stable; CIN, chromosomally instable; nCTx, neoadjuvant chemotherapy; TRG, tumor regression grades; Cis, cisplatin; Ox, oxaliplatin; 5-FU, 5-fluorouracil; Cap, capecitabine; Doc, docetaxel; Pac, paclitaxel; Epi, epirubicin; Others, combination of Cis/Ox with other agents or cross over between different treatment regimens; ${ }^{*}$ five patients without information for SMARCA2, SMARCA4, SMARCB1, ARID1A, ARID1B, or PBRM1; ${ }^{* *}$ four patients without information for SMARCA2, SMARCA4, SMARCB1, ARID1A, ARID1B, or PBRM1; italics values: Number of patients who received nCTx.

Response to preoperative chemotherapy was determined histopathologically and was classified into three tumor regression grades (TRGs): TRG1b, TRG2, and TRG3, which corresponded to $<10 \%, 10-50 \%$, and $>50 \%$ residual tumor cells [27]. Patients with TRG1b were classified as responders and with TRG2 and TRG3 as non-responders. Patients were treated with platinum/5-fluorouracil (5FU)-based chemotherapeutic regimes (Table 1). All surgical approaches included an abdominal D2-lymphadenectomy [28].

Follow-up data were obtained from the tumor data management of the University Hospital of Augsburg. Median follow-up was calculated by the inverse Kaplan-Meier method [29]. The primary endpoint of the study was overall survival (OS), which was defined as the time between the date of diagnosis and death by any cause.

The study was approved by the Institutional Review Board at the Ludwig-MaximiliansUniversity of Munich (reference: 20-0922) and was performed in accordance with the Declaration of Helsinki.

\subsection{Tissue Microarray Construction}

All eligible histological sections were first re-evaluated using a light microscope (Olympus, Shinjuku, Japan) to verify the diagnosis. Representative slides of each tumor were digitalized using a Pannoramic SCAN II scanner (3DHISTECH, Budapest, Hungary), and five areas, consisting of normal tissue $(1 \times)$, central tumor $(2 \times)$, and tumor invasion front $(2 \times)$, were selected. Based on the marked areas, formalin-fixed, paraffin-embedded (FFPE) tumor samples were subsequently automatically assembled into a tissue microarray (TMA) using a TMA Grandmaster (3DHISTECH, Budapest, Hungary) with a core size of $1 \mathrm{~mm}$.

\subsection{Immunohistochemistry and In Situ Hybridization}

Immunohistochemical staining was performed on $2 \mu \mathrm{m}$ sections from each TMA using primary antibodies listed in Supplementary Table S1. For PMS2, E-cadherin, CK7, CK20, CDX2, and EMA, a Ventana BenchMark ULTRA platform with an iVIEW DAB detection system was used (Roche, Mannheim, Germany). Staining for p53, SMARCA2, SMARCA4, SMARCB1, ARID1A, ARID1B, PBRM1, and MSH6 was performed on a BOND Rx platform with a BOND Polymer Refine Detection kit (Leica Biosystems, Nussloch, Germany). EBVpositive $\left(\mathrm{EBV}^{+}\right)$cases were identified by chromogenic in situ hybridization (EBER-CISH) likewise with the Ventana BenchMark ULTRA platform (Roche, Mannheim, Germany). Adequate controls were used for quality control of staining. 
The stained sections were again digitalized, and the evaluation was performed with 3DHISTECH Casviewer (3DHISTECH, Budapest, Hungary) by one pathologist (Bianca Grosser) and one trained researcher (Marie-Isabelle Glückstein.). Discrepant cases were discussed with a senior pathologist (Bruno Märkl), and a consensus was established. The investigators were blinded both to the clinicopathological data and outcome.

Immunohistochemical expression of SMARCA2, SMARCA4, SMARCB1, ARID1A, ARID1B, and PBRM1 was classified as retained, reduced, loss, or hybrid-loss [21]. A strong homogeneous nuclear staining of non-neoplastic cells served as internal control. Reduced expression was defined as homogenous, very weak, but still recognizable, staining compared to normal cells. Tumors with hybrid loss showed loss of expression only in a subset of cells. Specimens lacking strong staining in the background of normal cells were not assessed [21,30].

\subsection{TCGA Classification}

Tumors were classified in analogy to TCGA-classification [10] as proposed by Setia et al. and Ahn et al. [31,32] in $\mathrm{EBV}^{+}$, mismatch repair deficient (MMRD), genomically stable (GS), and chromosomally instable (CIN) cases. Cases that showed nuclear staining by EBER-CISH were considered $\mathrm{EBV}^{+}$. The presence of MMRD was stated in case of loss of nuclear expression of MSH6 or PMS2. GS cases were identified according to aberrant E-cadherin expression. E-cadherin was considered positive if membranous staining was present in more than 50\% of tumor cells [33]. Tumors were classified as CIN if an aberrant p53 expression pattern was present. p53 expression was considered aberrant if tumor cells showed complete loss of nuclear expression or if they showed staining with strong intensity in more than $60 \%$. Staining of less than $60 \%$ with weak to moderate intensity was considered a wild-type expression pattern [34,35]. Cases that did not meet the above criteria were designated as unclassifiable.

\subsection{Statistical Analysis}

Chi-squared tests were used for hypothesis testing of differences between the relative frequencies. Kaplan-Meier estimates of survival rates were compared by log rank tests. Relative risks were estimated by hazard ratios (HR) from Cox proportional hazard models. Statistical analyses were performed using SPSS, Version 24 (IBM Corp., Armonk, NY, USA) and R Version 4.0.3. Exploratory 5\% significance levels (two-tailed) were used for hypothesis testing.

\section{Results}

\subsection{Cohort}

The final cohort consisted of 477 patients with adenocarcinoma of the stomach or the gastroesophageal junction (Table 1). Of these, 130 patients received neoadjuvant chemotherapy and 347 underwent primary resection. The mean age of the cohort was 70.0 (range: 30.0-95.0) years, median follow up was 58.0 (range: 49.9-66.1) months; 52\% of patients died during the follow-up period. Detailed clinicopathological characteristics are shown in Table 1.

\subsection{SMARCA4 Expression}

Analyses showed complete loss of SMARCA4 expression in three cases $(0.6 \%)$. All tumors show an anaplastic solid and rhabdoid growth pattern (Figure 1), advanced T-stages, lymph node, and liver metastases. The median survival was eight (range: 7-12) months. Two patients received neoadjuvant chemotherapy and showed no response (TRG3). Two are subclassified as GS and one as CIN in analogy to the TCGA classification. All three cases were localized at the gastroesophageal junction (AEGII). Seven additional tumors $(1.4 \%)$ showed reduced SMARCA4 expression. In all three cases with SMARCA4 loss, the expression of ARID1A and SMARCB1 was intact, whereas SMARCA2 was reduced in two of them and completely lost in one case as well. In one case, tumor cells turned out to 
be negative with both CK7 and CK20, whereas vimentin was expressed with a distinct perinuclear dot-like pattern. In addition, there was no expression of CDX2, and EMA was expressed in all three cases with SMARCA4 loss.
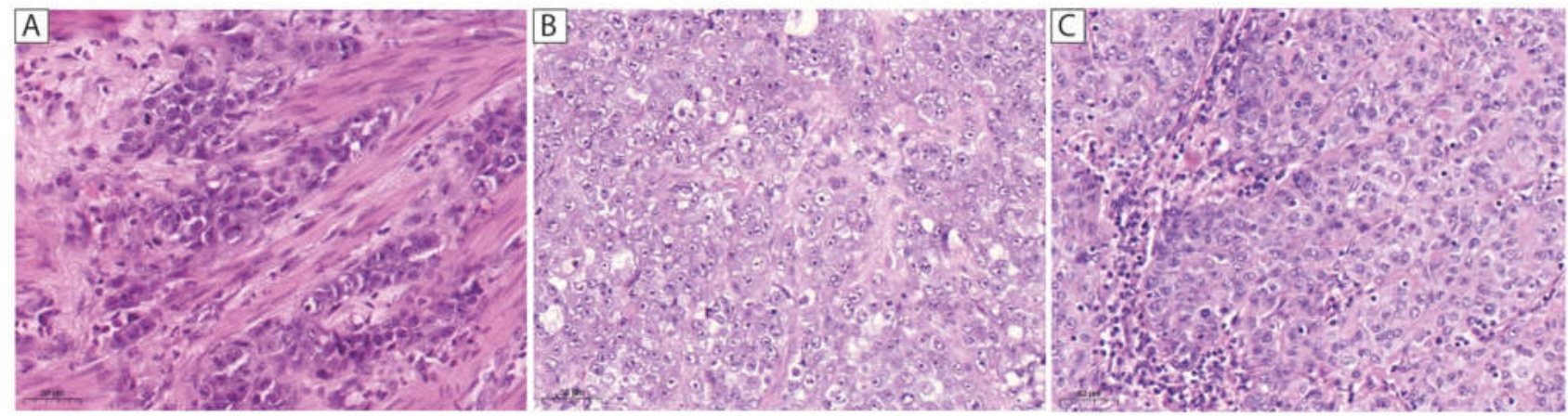

Figure 1. H\&E (hematoxylin and eosin) images of the three cases with complete loss of SMARCA4 expression. All three cases (A-C) show an anaplastic solid and rhabdoid growth pattern. (A) The tumor shows overexpression of p53, expression of CK7, CK20, CDX2, EMA, and no expression of vimentin. (B) In this case expression of SMARCA2 and E-cadherin is completely lost. CK7, CK20, and CDX2 are not expressed whereas vimentin and EMA are expressed. (C) This tumor shows complete loss of E-Cadherin, expression of CK20, CDX2, EMA and no expression of CK7 and vimentin. Scale bar $=50 \mu \mathrm{m}$.

\subsection{SMARCA2, SMARCB1, and ARID1A Expression}

Complete loss of ARID1A was observed in $59(12.5 \%)$ and reduced expression in 11 (2.3\%) cases. SMARCA2 expression was lost in $25(5.4 \%)$ tumors and reduced in $65(13.9 \%)$ cases. No case showed complete loss of SMARCB1. However, a heterogeneous expression pattern was detected in one case $(0.2 \%)$. In addition, five cases $(1.1 \%)$ showed reduced expression. Complete loss of PBRM1 occurred in 26 cases (5.5\%) for PBRM1 and nine cases (1.9\%) for ARID1B. Because both PBRM1 (31.4\%), and ARID1B (49.8\%) showed a very high proportion of cases with reduced expression, only cases with complete loss were designated as aberrant for consideration of the SWI/SNF status. Representative images of aberrant expression patterns are shown in Figure 2A-K and images of all cases with complete loss are presented in Supplementary Figure S1. In Figure 2M-R retained expression patterns of the proteins are shown.

As shown in Figure 2L, the largest overlap of aberrant expression can be seen between aberrant expression of SMARCA2 and ARID1A in 26 cases. One case showed simultaneous aberration of SMARCA4, SMARCA2, ARID1B, and PBRM1. No case with aberration of all the proteins involved in the SWI/SNF complex could be detected.

In cases of SMARCA2 loss, parallel loss of ARID1A occurred nine times. Seven of these nine cases showed intestinal type according to Laurén.

Regarding clinicopathological characteristics, complete loss of ARID1A was significantly associated with MMRD $(p<0.001)$ and aberrant expression with intestinal Laurén subtype $(p=0.014)$. Aberrant SMARCA2 expression was most frequently found in $\mathrm{EBV}^{+}$ tumors $(p<0.001)$, advanced tumor stages $(p=0.019)$, and patients with positive lymph nodes $(p=0.043)$. SMARCA2, $(p=0.160)$, ARID1A $(p=0.097)$, SMARCB1 $(p=0.930)$, PBRM1 $(p=0.520)$, and ARID1B $(p=0.490)$ expression showed no association with survival. Corresponding Kaplan-Meier curves are presented in Supplementary Figure S2. 

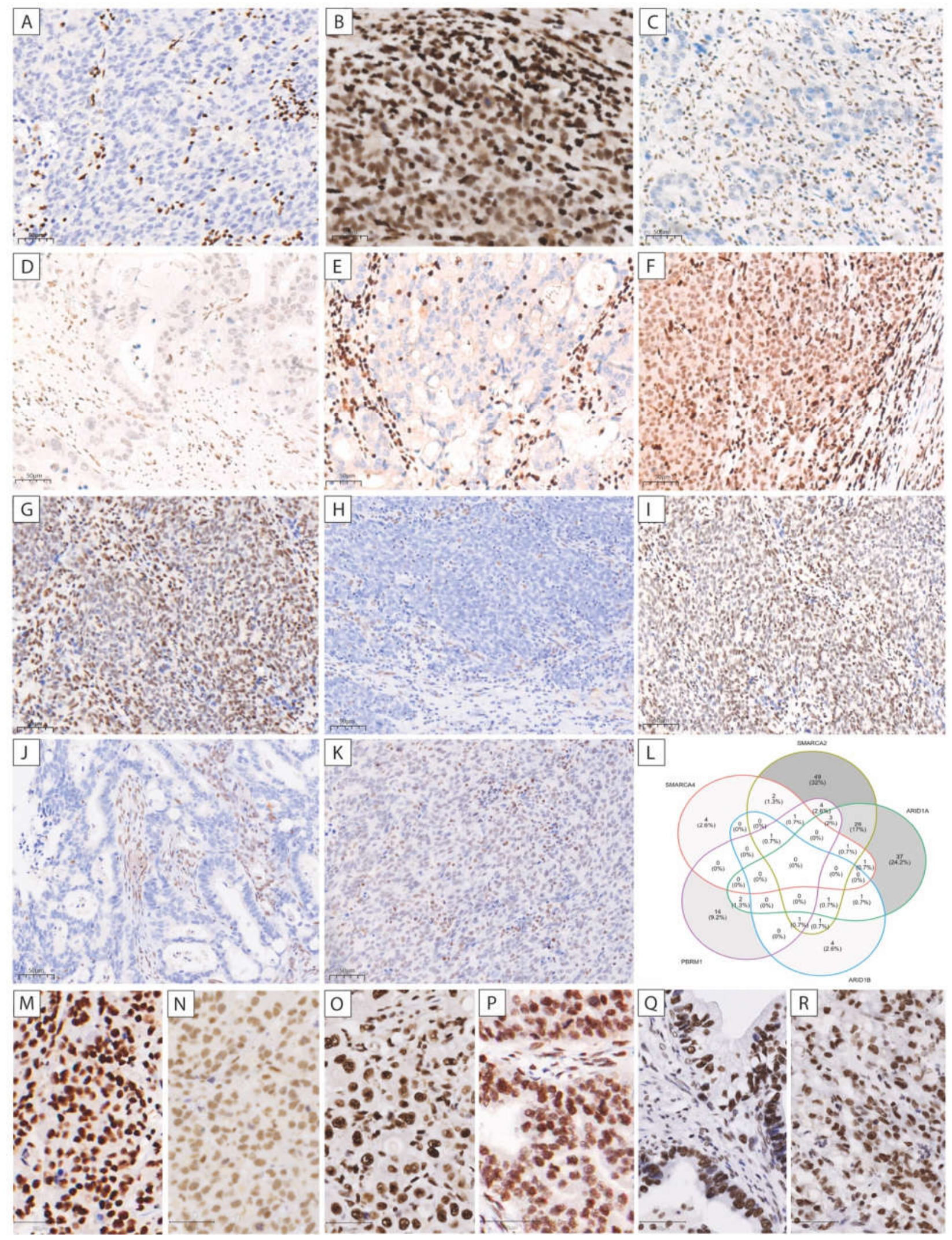

Figure 2. Representative images of immunohistochemical expression patterns of the proteins of the SWI/SNF complex. Complete loss of SMARCA4 (A), SMARCA2 (C), ARID1A (E), ARID1B (H), PBRM1 (J), and reduced expression of SMARCA4 (B), SMARCA2 (D), ARID1A (F), SMARCB1 (G), ARID1B (I), and PBRM1 (K) are shown. Venn-diagram of patients with loss or reduced expression of one of the proteins of the SWI/SNF complex (L). Retained expression patterns for SMARCA4 (M), SMARCA2 (N), ARID1A (O), SMARCB1 (P), ARID1B (Q), and PBRM1 (R) are presented. Scale bar $=50 \mu \mathrm{m}$. 


\subsection{SWI/SNF Status, Clinicopathologic Characteristics, and Survival}

In the following, patients are classified according to the expression of the SWI/SNF proteins. If any of the proteins SMARCA2, SMARCA4, SMARCB1, or ARID1A showed reduced expression or loss, the case was designated as SWI/SNF-aberrant (SWISNFab). The cases with reduced and lost expression were combined because no survival difference was observed between the two groups ( $p=0.452$ ) (Supplementary Figure S3). Only complete loss of PBRM1 or ARID1B expression was considered aberrant.

Cases with SWISNFab were associated with advanced T-stage and MMRD subtype (each $p<0.01)$ and in CTx patients with low chemotherapy response $(p=0.023)$. Other associations with clinicopathological characteristics are presented in Table 1.

In the overall cohort no significant survival difference regarding the SWI/SNF status could be observed ( $p=0.130$ ) (Figure 3A). SWISNFab patients had a median survival of 30.0 (range: 19.3-40.8) months compared to cases with intact expression with 44.0 (range: 30.6-57.4) months.
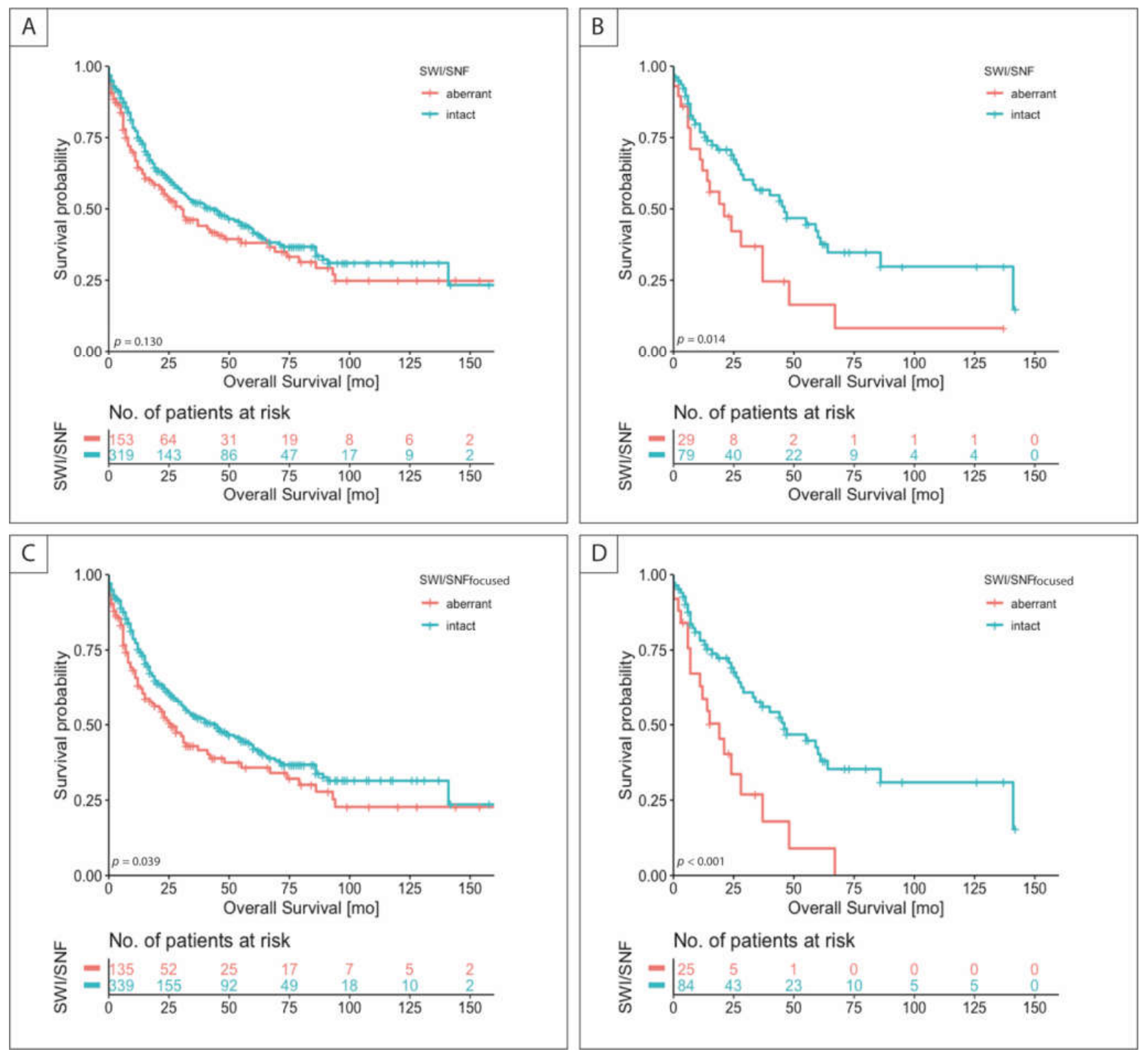

Figure 3. Discrimination of patient survival by SWI/SNF and SWI/SNFfocused status. Kaplan-Meier curves of patients with aberrant and intact proteins of the SWI/SNF complex are shown. SWI/SNF in all cases $(n=472)(\mathbf{A})$, in tumors classified as GS $(n=108)($ B $)$ and SWI/SNFfocused in all cases $(n=473)($ C), and in GS tumors $(n=109)(D) ; p$-value of log-rank test. 
In the subgroup analyses, the prognostic effect of SWISNFab was seen especially in tumors that were classified as GS in analogy to the TCGA classification $(p<0.014)$ (Figure 3B). SWISNFab patients had a median survival of 21.0 (range: 8.5-33.5) months compared to cases with intact expression with 46.0 (range: 23.8-68.2) months. In Cox regression analysis (Table 2), including known prognostic parameters, SWISNFab emerged as an independent prognostic factor for overall survival (HR 1.90, CI 1.04-3.50, $p=0.039$ ) in GS tumors. In the other subgroups, no survival difference was seen with respect to SWI/SNF status. The corresponding survival curves are presented in Supplementary Figure S3. The SWISNF expression status and survival of subgroups according to TCGA can be found in Supplementary Figure S4.

Table 2. Cox regression analyses of SWI/SNF status in all cases and in GS tumors.

\begin{tabular}{|c|c|c|c|c|c|c|c|c|}
\hline \multirow{3}{*}{$\begin{array}{l}\text { Variable } \\
\text { T-status }\end{array}$} & \multicolumn{4}{|c|}{ Overall Survival $(n=472)$} & \multicolumn{4}{|c|}{ Overall Survival $(n=108)$} \\
\hline & \multirow{2}{*}{$\begin{array}{c}\text { HR } \\
1.829\end{array}$} & \multicolumn{2}{|c|}{$95 \% \mathrm{CI}$} & \multirow{2}{*}{$\begin{array}{c}p \\
0.001\end{array}$} & \multirow{2}{*}{$\begin{array}{c}\text { HR } \\
1.898\end{array}$} & \multicolumn{2}{|c|}{$95 \%$ CI } & \multirow{2}{*}{$\begin{array}{c}p \\
0.186\end{array}$} \\
\hline & & 1.266 & 2.643 & & & 0.734 & 4.911 & \\
\hline N-status & 1.708 & 1.209 & 2.414 & 0.002 & 2.664 & 1.212 & 5.853 & 0.015 \\
\hline Age & 1.015 & 1.003 & 1.027 & 0.017 & 1.027 & 1.002 & 1.052 & 0.033 \\
\hline M-status & 2.517 & 1.820 & 3.480 & $<0.001$ & 2.045 & 1.027 & 4.073 & 0.042 \\
\hline R-status & 1.977 & 1.368 & 2.857 & $<0.001$ & 1.626 & 0.842 & 3.142 & 0.148 \\
\hline SWI/SNF & 1.218 & 0.898 & 1.651 & 0.205 & 1.904 & 1.035 & 3.503 & 0.039 \\
\hline TCGA & - & - & - & 0.440 & - & - & - & - \\
\hline $\mathrm{EBV}^{+}$ & 1.737 & 0.747 & 4.036 & 0.200 & - & - & - & - \\
\hline MSI & 1.753 & 0.781 & 3.934 & 0.173 & - & - & - & - \\
\hline GS & 2.047 & 0.921 & 4.552 & 0.079 & - & - & - & - \\
\hline CIN & 1.670 & 0.744 & 3.751 & 0.214 & - & - & - & - \\
\hline
\end{tabular}

CI, Confidence interval (95\%); TCGA, The Cancer Genome Atlas; EBV ${ }^{+}$, EBV-positive; MMRD, mismatch repair deficiency; GS, genomically stable; CIN, chromosomal instable.

\subsection{Determination of SWI/SNF Status Using a Focused Panel of Protein Expression}

To verify whether it is possible to reduce the panel for determining SWI/SNF status, only aberrant expression of SMARCA2, SMARCA4, SMARCB1, and ARID1A was considered and designated as SWI/SNFfocused.

Compared with SWI/SNF status, 18 patients were thus not classified as aberrant (Figure 2L). With regard to clinicopathologic characteristics, no essential difference was detected between SWI/SNF and SWI/SNFfocused (Supplementary Table S2).

As shown for SWI/SNFab, aberrant SWI/SNFfocused status emerged as an independent prognostic factor in GS tumors (HR 2.26, 95\% CI 1.19-4.28, $p=0.012$ ), and additionally also in the overall patient cohort (HR 1.42, 95\% CI 1.03-1.95, $p=0.03$ ) (Figure 3C,D, Supplementary Table S3).

\section{Discussion}

To the best of our knowledge, no study has evaluated the clinical relevance of the SWI/SNF complex in a large Western cohort of gastric cancer patients [21-24,36].

This study addressed this issue and analyzed the clinicopathological and prognostic relevance of the SWI/SNF complex in gastric adenocarcinomas with or without neoadjuvant CTx. We additionally investigated the association of the SWI/SNF complex with molecular subgroups in analogy to the TCGA.

The SWI/SNF complex has important functions in the mobilization of nucleosomes and consequently influences gene expression. Genes encoding subunits of the SWI/SNF complex are mutated in approximately $20 \%$ of all human tumors [18-20,37]. In our cohort, $32 \%$ of cases showed alteration of at least one of the subunits of the SWI/SNF complex, namely SMARCA2, SMARCA4, SMARCB1, ARID1A, PBRM1, and ARID1B.

Alterations of SMARCA4 occur in very low frequencies in solid tumors. We observed a loss of SMARCA4 expression in three cases $(0.6 \%)$. Similar frequencies have been observed in other studies in gastric, esophageal, and lung carcinomas [22,24,30,38]. All 
three tumors with SMARCA4 deficiency showed very similar, specific histopathologic features and were located at the gastroesophageal junction (AEGII). Additionally, they showed very adverse clinical characteristics and poor survival. The specific growth pattern and clinical significance have been described by Agaimy et al. [21] in two cases and Huang et al. [22] in six cases. As described by Agaimy et al. [21] the expression pattern of cytokeratins was different among the SMARCA4-deficient cases. In the case of SMARCA4 loss, epithelial membrane antigen (EMA) seems to be an adequate marker to proof epithelial differentiation, whereas vimentin showed only in one case, a typical perinuclear dot-like pattern [39]. Furthermore, we observed $>50 \%$ residual tumor (TRG3) in the two cases with SMARCA4 deficiency and preoperative CTx. As in adenocarcinomas of the lung and esophagus, no case with complete loss of SMARCB1 could be detected, suggesting that this subunit of the SWI/SNF complex does not play an overly important role in gastric carcinomas $[24,30,38]$.

SWI/SNF alteration within at least one of its subunits was an independent negative prognostic factor for overall survival. This is totally in line with a very recently published large Asian gastric cancer cohort study, where SWI/SNF was altered in 35\% of carcinomas and associated with a negative prognostic effect of altered SWI/SNF mainly in non-MSI/EBV diffuse type gastric carcinomas. Lacking data according to the molecular classification, this study could not further subclassify this non-MSI/EBV type [24]. Interestingly, we identified the GS group as mainly influenced by alterations of the SWI/SNF complex. The already-poor prognosis of this group that accounts for $23 \%$ of cases in our study was dramatically worsened in the SWSNFab group with a median survival time of 21 versus 46 months. In the GS subgroup $29(19 \%)$ cases showed altered SWI/SNF. In contrast to the Asian study, we did not observe a survival difference in the subgroup analyses according to Laurén subtypes [24]. There might be an overlap with the diffuse type as $83 \%$ of the carcinomas in our GS subgroup were classified as diffuse type. Regarding the relatively high percentage of SWI/SNF-deficient carcinomas and the poor prognosis especially in the GS subtype, there is urgent need for new therapeutic strategies.

For ARID1A-deficient cancer cells, Ogiwara et al. [40] showed that they express low levels of gluthation (GSH), which makes them specifically vulnerable to inhibition of the GSH metabolic pathway. Additionally, increased sensitivity of ARID1A-deficient cancer cells to treatment with small molecule inhibitor of the PI3K/AKT pathway or selective sensitivity of EZH2 inhibitors against ARID1A-deficient gastric cancer could be demonstrated [41-43]. EZH2 inhibitor tazemostat is currently investigated in ongoing clinical trials including SMARCA4-negative solid tumors [18]. The most promising potential therapeutic option so far seems to be the sensitivity to double-strand DNA breaks inducing agents like PARP inhibitors because of the impairment of the DNA damage checkpoint [44]. PARP inhibitors are currently evaluated in several ongoing clinical trials. Furthermore, Shorstova et al. [45] found SWI/SNF compromised cancers to be susceptible to bromodomain inhibitors. An allosteric inhibitor of SMARCA2 and SMARCA4 has demonstrated anti-proliferative activity in a mouse xenograft model of SMARCA4-mutant lung cancer [20]. Initial studies showed the potential efficiency of checkpoint inhibitors and promotion of anti-tumor immunity in SWI/SNF-deficient tumors [46,47]. Interestingly, SMARCB1-mutant rhabdoid tumors and SMARCA4-mutant small cell carcinoma of the ovary have an immune active microenvironment and are responsive to immune-checkpoint inhibition [18,48,49]. We could also observe a strong association of SWI/SNF deficiency with MMRD subtype, for which checkpoint-inhibition is already a therapeutic option $[50,51]$.

The analysis of a reduced panel of proteins to determine the SWI/SNF status proved to be almost equivalent in terms of determining the prognosis. Especially with regard to a possible determination of SWI/SNF status in routine diagnostics, it seems reasonable to limit the determination of SWI/SNF status in gastric cancer to SMARCA4, SMARCA2, and ARID1A.

Despite the comprehensive analysis of a large cohort, our study has limitations, which are mainly related to its retrospective nature. Our study has to be considered as 
an exploratory analysis and the results have to be validated in independent prospective cohorts. To elucidate the underlying molecular mechanisms of the alteration, we identified on the protein expression level, that sophisticated genetic and epigenetic investigations are necessary.

\section{Conclusions}

In summary, the expression of SMARCB1, does not appear to be of major importance in gastric carcinoma. The determination of SWI/SNF status with analyses of SMARCA2, SMARCA4, and ARID1A could be considered in routine practice, especially in the GS subgroup according to TCGA, to identify patients who might potentially benefit from new therapeutic alternatives.

Supplementary Materials: The following are available online at https:/ / www.mdpi.com/article / 10.3390/cancers13153894/s1, Figure S1: SWI/SNF expression status and survival in subgroups according to TCGA; Figure S2: Kaplan-Meier curves of the patients with aberrant, reduced, and intact expression of ARID1A (A), SMARCA2 (B), SMARCA4 (C), SMARCB1 (D), PBRM1 (E), and ARID1B (F) are shown; Figure S3: Survival in cases with complete and reduced expression of SWI/SNFfocused status; Figure S4: SWI/SNF expression status and survival in subgroups according to TCGA; Table S1: Antibodies and dilutions; Table S2: Clinicopathological characteristics and SWI/SNFfocused status; Table S3: Cox regression analysis of SWI/SNFfocused status.

Author Contributions: Conceptualization, B.M., B.G., M.-I.G., and S.D.; formal analysis, M.-I.G., B.G., and T.T.A.; investigation, B.G., M.-I.G., S.D., D.V., and G.S.; writing-original draft preparation, M.-I.G. and B.G.; writing-review and editing, B.M., B.G., M.-I.G., S.D., and A.A.; visualization, M.-I.G. and B.G.; supervision-B.M. and G.M. All authors have read and agreed to the published version of the manuscript.

Funding: The study received no external funding.

Institutional Review Board Statement: The study was approved by the institutional Review Boards at the Ludwig-Maximilians-University of Munich (reference: 20-0922, 17 December 2020) and was performed in accordance with the Declaration of Helsinki.

Informed Consent Statement: Patient consent was waived due to the retrospective study type with anonymization of patients.

Data Availability Statement: The datasets generated during the current work are available from the corresponding author on reasonable request.

Acknowledgments: The authors are grateful to Jenny Müller, Andrea Seuser, and Christian Beul for excellent technical assistance.

Conflicts of Interest: The authors have no conflict of interest to declare that are relevant to the content of this article.

\section{References}

1. Bray, F.; Ferlay, J.; Soerjomataram, I.; Siegel, R.L.; Torre, L.A.; Jemal, A. Global cancer statistics 2018: GLOBOCAN estimates of incidence and mortality worldwide for 36 cancers in 185 countries. CA Cancer J. Clin. 2018, 68, 394-424. [CrossRef]

2. Brierley, J.D.; Gospodarowicz, M.K.; Wittekind, C. TNM Classification of Malignant Tumours, 8th ed.; John Wiley \& Sons Ltd.: Chichester, UK, 2017; pp. 115-119.

3. In, H.; Solsky, I.; Palis, B.; Langdon-Embry, M.; A Ajani, J.; Sano, T. Validation of the 8th Edition of the AJCC TNM Staging System for Gastric Cancer using the National Cancer Database. Ann. Surg. Oncol. 2017, 24, 3683-3691. [CrossRef] [PubMed]

4. Shiraishi, N.; Sato, K.; Yasuda, K.; Inomata, M.; Kitano, S. Multivariate prognostic study on large gastric cancer. J. Surg. Oncol. 2007, 96, 14-18. [CrossRef]

5. Chen, Y.-C.; Fang, W.-L.; Wang, R.-F.; Liu, C.-A.; Yang, M.-H.; Lo, S.-S.; Wu, C.-W.; Li, A.F.-Y.; Shyr, Y.-M.; Huang, K.-H. Clinicopathological Variation of Lauren Classification in Gastric Cancer. Pathol. Oncol. Res. 2016, 22, 197-202. [CrossRef]

6. Berlth, F. Pathohistological classification systems in gastric cancer: Diagnostic relevance and prognostic value. World J. Gastroenterol. 2014, 20, 5679-5684. [CrossRef]

7. Liu, K.; Zhang, W.; Chen, X.; Chen, X.; Yang, K.; Zhang, B.; Chen, Z.; Zhou, Z.; Hu, J. Comparison on Clinicopathological Features and Prognosis Between Esophagogastric Junctional Adenocarcinoma (Siewert II/III Types) and Distal Gastric Adenocarcinoma. Medicine 2015, 94, e1386. [CrossRef] 
8. Cunningham, D.; Allum, W.H.; Stenning, S.P.; Thompson, J.N.; Van De Velde, C.J.; Nicolson, M.; Scarffe, J.H.; Lofts, F.J.; Falk, S.J.; Iveson, T.J.; et al. Perioperative Chemotherapy versus Surgery Alone for Resectable Gastroesophageal Cancer. N. Engl. J. Med. 2006, 355, 11-20. [CrossRef]

9. Allemani, C.; Matsuda, T.; Di Carlo, V.; Harewood, R.; Matz, M.; Nikšić, M.; Bonaventure, A.; Valkov, M.; Johnson, C.J.; Estève, J.; et al. Global surveillance of trends in cancer survival 2000-14 (CONCORD-3): Analysis of individual records for 37513025 patients diagnosed with one of 18 cancers from 322 population-based registries in 71 countries. Lancet 2018, 391, $1023-1075$. [CrossRef]

10. Cancer Genome Atlas Research Network. The Cancer Genome Atlas Research Network Comprehensive molecular characterization of gastric adenocarcinoma. Nat. Cell Biol. 2014, 513, 202-209. [CrossRef]

11. Cristescu, R.; Lee, J.; Nebozhyn, M.; Kim, K.-M.; Ting, J.C.; Wong, S.S.; Liu, J.; Yue, Y.G.; Wang, J.; Yu, K.; et al. Molecular analysis of gastric cancer identifies subtypes associated with distinct clinical outcomes. Nat. Med. 2015, 21, 449-456. [CrossRef] [PubMed]

12. Zhou, F.; Li, N.; Jiang, W.; Hua, Z.; Xia, L.; Wei, Q.; Wang, L. Prognosis significance of HER-2/neu overexpression/amplification in Chinese patients with curatively resected gastric cancer after the ToGA clinical trial. World J. Surg. Oncol. 2012, 10, 274. [CrossRef]

13. Böger, C.; Behrens, H.-M.; Mathiak, M.; Krüger, S.; Kalthoff, H.; Röcken, C. PD-L1 is an independent prognostic predictor in gastric cancer of Western patients. Oncotarget 2016, 7, 24269-24283. [CrossRef] [PubMed]

14. Muro, K.; Chung, H.; Shankaran, V.; Geva, R.; Catenacci, D.; Gupta, S.; Eder, J.P.; Golan, T.; Le, D.T.; Burtness, B.; et al. Pembrolizumab for patients with PD-L1-positive advanced gastric cancer (KEYNOTE-012): A multicentre, open-label, phase 1b trial. Lancet Oncol. 2016, 17, 717-726. [CrossRef]

15. Baniak, N.; Senger, J.-L.; Ahmed, S.; Kanthan, S.C.; Kanthan, R. Gastric biomarkers: A global review. World J. Surg. Oncol. 2016, 14, 1-14. [CrossRef] [PubMed]

16. Ferreira, P.; Oliveira, M.J.; Beraldi, E.; Mateus, A.R.; Nakajima, T.; Gleave, M.; Yokota, J.; Carneiro, F.; Huntsman, D.; Seruca, R.; et al. Loss of functional E-cadherin renders cells more resistant to the apoptotic agent taxol in vitro. Exp. Cell Res. 2005, 310, 99-104. [CrossRef]

17. Eberhart, C.G.; A Rubens, J. SWI/SNF complex differences promote cellular heterogeneity in rhabdoid tumors. Neuro-Oncology 2020, 22, 741-742. [CrossRef] [PubMed]

18. Mittal, P.; Roberts, C.W.M. The SWI/SNF complex in cancer - biology, biomarkers and therapy. Nat. Rev. Clin. Oncol. 2020, 17, 435-448. [CrossRef] [PubMed]

19. Masliah-Planchon, J.; Bieche, I.; Guinebretière, J.-M.; Bourdeaut, F.; Delattre, O. SWI/SNF Chromatin Remodeling and Human Malignancies. Annu. Rev. Pathol. Mech. Dis. 2015, 10, 145-171. [CrossRef]

20. Papillon, J.P.N.; Nakajima, K.; Adair, C.D.; Hempel, J.; Jouk, A.O.; Karki, R.G.; Mathieu, S.; Moebitz, H.; Ntaganda, R.; Smith, T.; et al. Discovery of Orally Active Inhibitors of Brahma Homolog (BRM)/SMARCA2 ATPase Activity for the Treatment of Brahma Related Gene 1 (BRG1)/SMARCA4-Mutant Cancers. J. Med. Chem. 2018, 61, 10155-10172. [CrossRef]

21. Agaimy, A.; Daum, O.; Märkl, B.; Lichtmannegger, I.; Michal, M.; Hartmann, A. SWI/SNF Complex-deficient Undifferentiated/Rhabdoid Carcinomas of the Gastrointestinal Tract. Am. J. Surg. Pathol. 2016, 40, 544-553. [CrossRef]

22. Huang, S.; Ng, K.; Yeh, T.; Cheng, C.; Chen, M.; Chao, Y.; Chuang, H.; Liu, Y.; Chen, T. The clinicopathological and molecular analysis of gastric cancer with altered SMARCA4 expression. Histopathology 2020, 77, 250-261. [CrossRef]

23. Tessier-Cloutier, B.; Schaeffer, D.F.; Bacani, J.; E Marginean, C.; Kalloger, S.; Köbel, M.; Lee, C. Loss of switch/sucrose nonfermenting complex protein expression in undifferentiated gastrointestinal and pancreatic carcinomas. Histopathology 2020, 77, 46-54. [CrossRef]

24. Huang, S.-C.; Ng, K.-F.; Chang, I.Y.-F.; Chang, C.-J.; Chao, Y.-C.; Chang, S.-C.; Chen, M.-C.; Yeh, T.-S.; Chen, T.-C. The clinicopathological significance of SWI/SNF alterations in gastric cancer is associated with the molecular subtypes. PLoS ONE 2021, 16, e0245356. [CrossRef]

25. Liu, J.; Lichtenberg, T.; Hoadley, K.A.; Poisson, L.M.; Lazar, A.J.; Cherniack, A.D.; Kovatich, A.J.; Benz, C.C.; Levine, D.A.; Lee, A.V.; et al. An Integrated TCGA Pan-Cancer Clinical Data Resource to Drive High-Quality Survival Outcome Analytics. Cell 2018, 173, 400-416.e11. [CrossRef] [PubMed]

26. Siewert, J.R.; Stein, H.J. Classification of adenocarcinoma of the oesophagogastric junction. BJS 2003, 85, 1457-1459. [CrossRef]

27. Becker, K.; Mueller, J.D.; Schulmacher, C.; Ott, K.; Fink, U.; Busch, R.; Böttcher, K.; Siewert, J.R.; Höfler, H. Histomorphology and grading of regression in gastric carcinoma treated with neoadjuvant chemotherapy. Cancer 2003, 98, 1521-1530. [CrossRef]

28. Songun, I.; Putter, H.; Kranenbarg, E.M.-K.; Sasako, M.; van de Velde, C.J. Surgical treatment of gastric cancer: 15-year follow-up results of the randomised nationwide Dutch D1D2 trial. Lancet Oncol. 2010, 11, 439-449. [CrossRef]

29. Shuster, J.J. Median follow-up in clinical trials. J. Clin. Oncol. 1991, 9, 191-192. [CrossRef]

30. Herpel, E.; Rieker, R.J.; Dienemann, H.; Muley, T.; Meister, M.; Hartmann, A.; Warth, A.; Agaimy, A. SMARCA4 and SMARCA2 deficiency in non-small cell lung cancer: Immunohistochemical survey of 316 consecutive specimens. Ann. Diagn. Pathol. 2017, 26, 47-51. [CrossRef]

31. Ahn, S.; Lee, S.-J.; Kim, Y.; Kim, A.; Shin, N.; Choi, K.U.; Lee, C.-H.; Huh, G.Y.; Kim, K.-M.; Setia, N.; et al. High-throughput Protein and mRNA Expression-based Classification of Gastric Cancers Can Identify Clinically Distinct Subtypes, Concordant With Recent Molecular Classifications. Am. J. Surg. Pathol. 2017, 41, 106-115. [CrossRef] [PubMed] 
32. Setia, N.; Agoston, A.T.; Han, H.S.; Mullen, J.T.; Duda, D.G.; Clark, J.W.; Deshpande, V.; Mino-Kenudson, M.; Srivastava, A.; Lennerz, J.K.; et al. A protein and mRNA expression-based classification of gastric cancer. Mod. Pathol. 2016, 29, 772-784. [CrossRef]

33. Bronsert, P.; Kohler, I.; Timme, S.; Kiefer, S.; Werner, M.; Schilling, O.; Vashist, Y.; Makowiec, F.; Brabletz, T.; Hopt, U.T.; et al. Prognostic significance of Zinc finger E-box binding homeobox 1 (ZEB1) expression in cancer cells and cancer-associated fibroblasts in pancreatic head cancer. Surgery 2014, 156, 97-108. [CrossRef]

34. Köbel, M.; Piskorz, A.M.; Lee, S.; Lui, S.; Lepage, C.; Marass, F.; Rosenfeld, N.; Mes-Masson, A.-M.; Brenton, J.D. Optimized p53 immunohistochemistry is an accurate predictor of TP53 mutation in ovarian carcinoma. J. Pathol. Clin. Res. 2016, 2, 247-258. [CrossRef]

35. Grosser, B.; Kohlruss, M.; Slotta-Huspenina, J.; Jesinghaus, M.; Pfarr, N.; Steiger, K.; Novotny, A.; Gaida, M.M.; Schmidt, T.; Hapfelmeier, A.; et al. Impact of Tumor Localization and Molecular Subtypes on the Prognostic and Predictive Significance of p53 Expression in Gastric Cancer. Cancers 2020, 12, 1689. [CrossRef] [PubMed]

36. Agaimy, A.; Rau, T.T.; Hartmann, A.; Stoehr, R. SMARCB1 (INI1)-negative Rhabdoid Carcinomas of the Gastrointestinal Tract. Am. J. Surg. Pathol. 2014, 38, 910-920. [CrossRef]

37. Guerrero-Martínez, J.A.; Reyes, J.C. High expression of SMARCA4 or SMARCA2 is frequently associated with an opposite prognosis in cancer. Sci. Rep. 2018, 8, 2043. [CrossRef]

38. Schallenberg, S.; Bork, J.; Essakly, A.; Alakus, H.; Buettner, R.; Hillmer, A.M.; Bruns, C.; Schroeder, W.; Zander, T.; Loeser, H.; et al. Loss of the SWI/SNF-ATPase subunit members SMARCF1 (ARID1A), SMARCA2 (BRM), SMARCA4 (BRG1) and SMARCB1 (INI1) in oesophageal adenocarcinoma. BMC Cancer 2020, 20, 12. [CrossRef] [PubMed]

39. Nagtegaal, I.D.; Odze, R.D.; Klimstra, D.; Paradis, V.; Rugge, M.; Schirmacher, P.; Washington, K.M.; Carneiro, F.; Cree, I.A.; the WHO Classification of Tumours Editorial Board. The 2019 WHO classification of tumours of the digestive system. Histopathology 2019, 76, 182-188. [CrossRef] [PubMed]

40. Ogiwara, H.; Takahashi, K.; Sasaki, M.; Kuroda, T.; Yoshida, H.; Watanabe, R.; Maruyama, A.; Makinoshima, H.; Chiwaki, F.; Sasaki, H.; et al. Targeting the Vulnerability of Glutathione Metabolism in ARID1A-Deficient Cancers. Cancer Cell 2019, 35, 177-190.e8. [CrossRef]

41. Samartzis, E.P.; Gutsche, K.; Dedes, K.J.; Fink, D.; Stucki, M.; Imesch, P. Loss of ARID1A expression sensitizes cancer cells to PI3Kand AKT-inhibition. Oncotarget 2014, 5, 5295-5303. [CrossRef]

42. Bitler, B.; Aird, K.M.; Garipov, A.; Li, H.; Amatangelo, M.; Kossenkov, A.V.; Schultz, D.C.; Liu, Q.; Shih, I.-M.; Conejo-Garcia, J.; et al. Synthetic lethality by targeting EZH2 methyltransferase activity in ARID1A-mutated cancers. Nat. Med. 2015, 21, 231-238. [CrossRef] [PubMed]

43. Yamada, L.; Saito, M.; Min, A.K.T.; Saito, K.; Ashizawa, M.; Kase, K.; Nakajima, S.; Onozawa, H.; Okayama, H.; Endo, H.; et al. Selective sensitivity of EZH2 inhibitors based on synthetic lethality in ARID1A-deficient gastric cancer. Gastric Cancer 2021, 24, 60-71. [CrossRef] [PubMed]

44. Shen, J.; Peng, Y.; Wei, L.; Zhang, W.; Yang, L.; Lan, L.; Kapoor, P.; Ju, Z.; Mo, Q.; Shih, I.-M.; et al. ARID1A Deficiency Impairs the DNA Damage Checkpoint and Sensitizes Cells to PARP Inhibitors. Cancer Discov. 2015, 5, 752-767. [CrossRef]

45. Shorstova, T.; Marques, M.; Su, J.; Johnston, J.; Kleinman, C.L.; Hamel, N.; Huang, S.; Alaoui-Jamali, M.A.; Foulkes, W.D.; Witcher, M. SWI/SNF-Compromised Cancers Are Susceptible to Bromodomain Inhibitors. Cancer Res. 2019, 79, 2761-2774. [CrossRef]

46. Zhou, M.; Yuan, J.; Deng, Y.; Fan, X.; Shen, J. Emerging role of SWI/SNF complex deficiency as a target of immune checkpoint blockade in human cancers. Oncogenesis 2021, 10, 1-8. [CrossRef]

47. Soldi, R.; Halder, T.G.; Weston, A.; Thode, T.; Drenner, K.; Lewis, R.; Kaadige, M.R.; Srivastava, S.; Ampanattu, S.D.; Del Villar, R.R.; et al. The novel reversible LSD1 inhibitor SP-2577 promotes anti-tumor immunity in SWItch/Sucrose-NonFermentable (SWI/SNF) complex mutated ovarian cancer. PLoS ONE 2020, 15, e0235705. [CrossRef]

48. Leruste, A.; Tosello, J.; Ramos, R.N.; Tauziède-Espariat, A.; Brohard, S.; Han, Z.-Y.; Beccaria, K.; Andrianteranagna, M.; Caudana, P.; Nikolic, J.; et al. Clonally Expanded T Cells Reveal Immunogenicity of Rhabdoid Tumors. Cancer Cell 2019, 36, 597-612.e8. [CrossRef]

49. Jelinic, P.; Ricca, J.; Van Oudenhove, E.; Olvera, N.; Merghoub, T.; A Levine, D.; Zamarin, D. Immune-Active Microenvironment in Small Cell Carcinoma of the Ovary, Hypercalcemic Type: Rationale for Immune Checkpoint Blockade. J. Natl. Cancer Inst. 2018, 110, 787-790. [CrossRef] [PubMed]

50. Kim, S.T.; Cristescu, R.; Bass, A.J.; Kim, K.-M.; Odegaard, J.I.; Kim, K.; Liu, X.Q.; Sher, X.; Jung, H.; Lee, M.; et al. Comprehensive molecular characterization of clinical responses to PD-1 inhibition in metastatic gastric cancer. Nat. Med. 2018, 24, 1449-1458. [CrossRef]

51. Fuchs, C.S.; Özgüroğlu, M.; Bang, Y.-J.; Di Bartolomeo, M.; Mandalà, M.; Ryu, M.-H.; Fornaro, L.; Olesinski, T.; Caglevic, C.; Chung, H.C.; et al. Pembrolizumab versus paclitaxel for previously treated patients with PD-L1-positive advanced gastric or gastroesophageal junction cancer (GC): Update from the phase III KEYNOTE-061 trial. J. Clin. Oncol. 2020, 38, 4503. [CrossRef] 